\title{
The Common Complications after Septoplasty and Septorhinoplasty: A Report in a Series of 127 Cases
}

\author{
Abdullah D. Alotaibi \\ Department of Otolaryngology Head and Neck Surgery, College of Medicine, Hail University, Hail, KSA \\ Email: adakheel505@gmail.com
}

How to cite this paper: Alotaibi, A.D. (2017) The Common Complications after Septoplasty and Septorhinoplasty: A Report in a Series of 127 Cases. International Journal of Otolaryngology and Head \& Neck Surgery, 6, 71-78.

https://doi.org/10.4236/ijohns.2017.66010

Received: November 11, 2017

Accepted: November 27, 2017

Published: November 30, 2017

Copyright $\odot 2017$ by author and Scientific Research Publishing Inc.

This work is licensed under the Creative

Commons Attribution International

License (CC BY 4.0).

http://creativecommons.org/licenses/by/4.0/

Open Access

\begin{abstract}
Background: There are many complications associated with septoplasty, which chiefly depend on the procedure performed. The aim of this study was to find out the common complications after Septoplasty and Septorhinoplasty in a series of 127 Saudi patients undergoing Septoplasty or Septorhinoplasty in Northern Saudi Arabia. Methodology: This study included a series of $127 \mathrm{pa}-$ tients presented with nasal blockage and subsequently undergone Septoplasty and /or Septorhinoplasty. Results: Complications were determined in 69/127 (54.3\%) of the patients. The most common complication after three months of septoplasty or Septorhinoplasty was the change in the shape of the nose, which represented 57 (82.6\%) out of 69 patients with complications. Bleeding was experienced in 8 patients of whom 5/8 (62.5\%) were males and $3 / 8$ (37.5\%) were females. Conclusion: Nose deformities are the most common complication associated with Septoplasty or Septorhinoplasty in Northern Saudi Arabia. The risks for Septoplasty or Septorhinoplasty complications can be condensed with accumulative experience.
\end{abstract}

\section{Keywords}

Septoplasty, Septorhinoplasty, Deviated Nasal Septum, Saudi Arabia

\section{Introduction}

Nasal obstruction is ordinarily demarcated as patient discomfort manifested as a sensation of inadequate airflow via the nose [1]. The cause of nasal obstruction is usually divided into mucosal and anatomical reasons. Nasal septal deviation (NSD) is the most common anatomical source of nasal blockade [2]. 
Septoplasty is a surgical method that aims to straighten out the deviated nasal septum (DNS). Septoplasty should not be mixed up with Septorhinoplasty, which is a surgical technique that addresses both the nasal septum as well as other structures of the nose [3]. Surgical amendment of the DNS is the most common ear, nose and throat (ENT) operation in adults [4]. Yearly Septoplasty rates, yet, vary worldwide [5] [6] [7].

There are several complications accompanying Septoplasty. The most common complications of Septoplasty are deformities, infections, and perforations. The special effects of each of these complications, however, can be very dissimilar. Displacements and deformities of the septum may result not only in a compromised airway but also in observable deformities of the entire nasal base and dorsum. A patient who experienced Septoplasty can be "stigmatized". Infections may lead not only to septal abscess but also to endocranial complications such as meningitis or septicemia with endocarditis. Long-lasting perforations of the nasal septum can result in important symptoms if they are situated in the anterior part of the nose. In addition to these major types of complications there are numerous others, from smell disorders to blindness [8].

The external rhinoplasty methodology was verified to be very useful in the course of septal perforation repair particularly in large and posteriorly situated perforations and in cases where the caudal septal cartilage was formerly resected. It was established that septal perforation repair can be safely combined with rhinoplasty and that some of the routine rhinoplasty maneuvers, such as medial osteotomies and dorsal lowering, could even simplify the procedure of septal perforation repair [9].

There is a paucity of literature regarding the complications associated with Septoplasty or Septorhinoplasty from Saudi Arabia. Therefore the aim of the present study was to find out the common complications after Septoplasty and Septorhinoplasty in a series of 127 Saudi patients undergoing Septoplasty or Septorhinoplasty in Northern Saudi Arabia.

\section{Materials and Methods}

This study involved a series of 127 patients presented with nasal blockage and subsequently undergone septoplasty and/or Septorhinoplasty. Archives related to all patients were retrieved from ENT department, King Khalid hospital in Hail, Northern Saudi Arabia. Only adults over 18 years of age were contained within the study. Patients' medical records were examined, and patients with a history of cranial and facial trauma or bone deformity (except DNS), and patients with a mass in the nasal cavity were excluded from the study. A number of nasal obstruction clinical presentations were recorded including nasal blockage. Demographical characteristics including; age, gender and residence were also recorded.

The different clinical complications were evaluated after three months of septoplasty and/or Septorhinoplasty. 


\subsection{Ethical Consent}

Our study protocol was conformed according to the 2013 Declaration of Helsin$\mathrm{ki}$ and this study was approved by ethics committee of College of Medicine, University of Hail, Saudi Arabia.

\subsection{Statistical Analysis}

Statistical analysis was performed using SPSS software for Windows (version 16.0, SPSS Inc., Chicago, IL, USA). Categorical variables are given as frequencies and percentages, and continuous variables. For all statistical comparisons, a $\mathrm{p}$ value below 0.05 was considered statistically significant.

\section{Results}

This study investigated records of 127 patients selected for Septoplasty due to several complaints associated with nasal blockage. Out of 127 patients, 96/127 (75.6\%) were males and 31/127 (24.4\%) were females, giving males' females' ration of 1.00 to 3.10 . The ages of the study subjects were ranging from 18 to 42 years with a mean age of 26.4 years old. The majority of the patients were at age group 21 - 25 years representing 42/127 (33\%), followed by age ranges, 26 - 30 years, $<20$ years, $31-35$ and 36+ representing 28/127 (22\%), 25/127 (20\%), $18 / 127(14 \%)$ and 14/127 (11\%), respectively. For males, the majority were at age group 21 - 25 years followed by 26 - 30 and 31 - 35 years constituting 29, 26 and 16 patients respectively. For females, the majority were at age group $21-25$ years followed by age group $<20$ years representing 13 and 11 patients in this order as indicated in Table 1, Figure 1.

With regard to the complications after Septoplasty, 58/127 (45.7\%) were free of complications of whom $42 / 58$ (72.4\%) were males and 16/58 (27.6\%) were females. Complications were determined in $69 / 127$ (54.3\%) of the patients. The most common complication after three months of Septoplasty was the change in

Table 1. Distribution of patients by age, initial diagnosis and sex.

\begin{tabular}{ccccc}
\hline Variable & Category & Males & Females & Total \\
\hline Age & $<20$ years & 14 & 11 & 25 \\
& $21-25$ & 29 & 13 & 42 \\
& $26-30$ & 26 & 2 & 28 \\
& $31-35$ & 16 & 2 & 18 \\
& $36+$ & 11 & 3 & 14 \\
Total & 96 & 31 & 127 \\
& DNS & & & 109 \\
& Yes & 85 & 24 & 127 \\
\hline
\end{tabular}




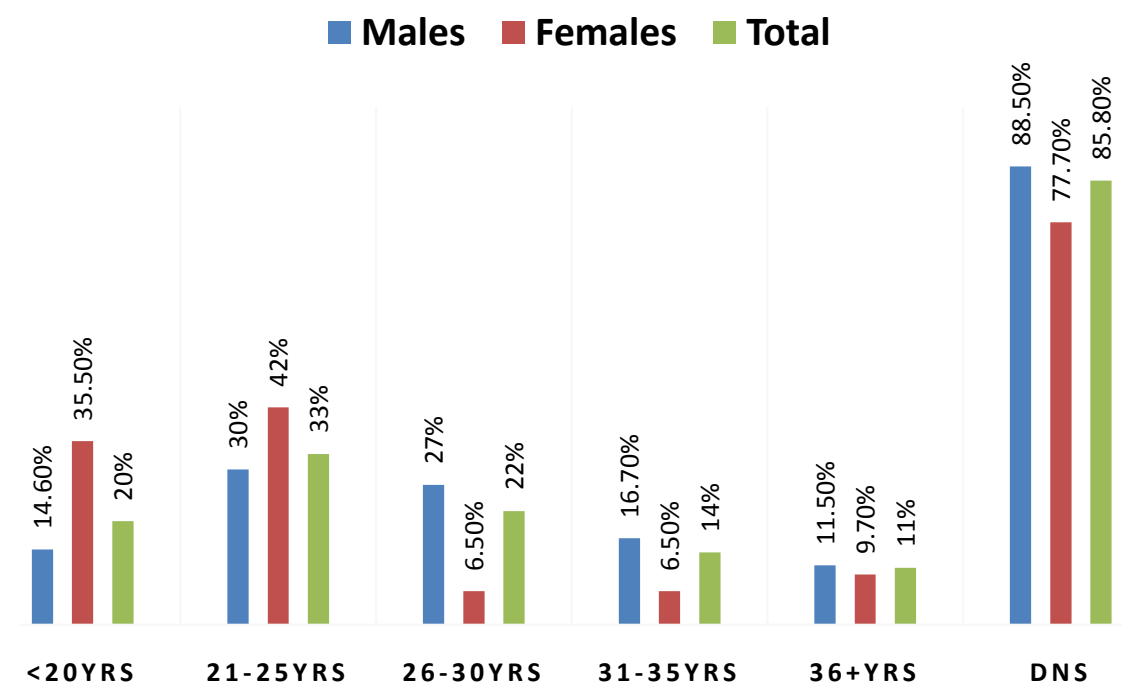

Figure 1. Description of patients by age, initial diagnosis and sex.

the shape of the nose, which represent 57 (82.6\%) out of 69 patients with complications. Out of 57 patients with nose's shape changes, 46/57 (80.7\%) were males and the remaining 11/57 (19.3\%) were females. Bleeding was experienced in 8 patients of whom 5/8 (62.5\%) were males and 3/8 (37.5\%) were females. Scar formation was identified in one male and one female. Inflammation was identified in one male, hence, loss of smell was identified in one female, as indicated in Table 2, Figure 2.

The distribution of patients by age, operation done and nasal blockage by complications after three months of septoplasty was summarized in Table 3. Most of the changes in the shape of the nose were observed in age group $21-25$ years followed by 26 - 30 years constituting 18/57 (31.6\%) and 11/57 (19.3\%) correspondingly. Bleeding was predominantly observed in age group $21-25$ years followed by $36+$ years representing $5 / 8(62.5 \%)$ and $2 / 8(25 \%)$ in this order. With regard to surgical procedure done, out of 103 septoplasty, change of nose shape and bleeding were observed in 41/103 (39.8\%) and 7/103 (6.8\%) respectively. Out of 24 Septorhinoplasty cases, change of nose shape was observed in 16/24 (66.7\%). Moreover, nasal blockage was more frequently observed among those with the change of nose shape, as indicated in Table 3, Figure 3.

\section{Discussion}

NSD is a widespread problem that can have substantial quality of life consequences. Septoplasty is usually done to offer qualitative and quantitative advantage to those with nasal blockage due to NSD. Though a standard, basic procedure is frequently satisfactory for patients with mild to moderate mid to posterior NSD, distinctive challenges arise with caudal NSD.

In the present study several complications that might be associated with Septoplasty or Septorhinoplasty have been investigated. The complications were assessed after three month of the surgical intercession. The overall assessment of 
Table 2. Distribution of patients by complications after three months of septoplasty.

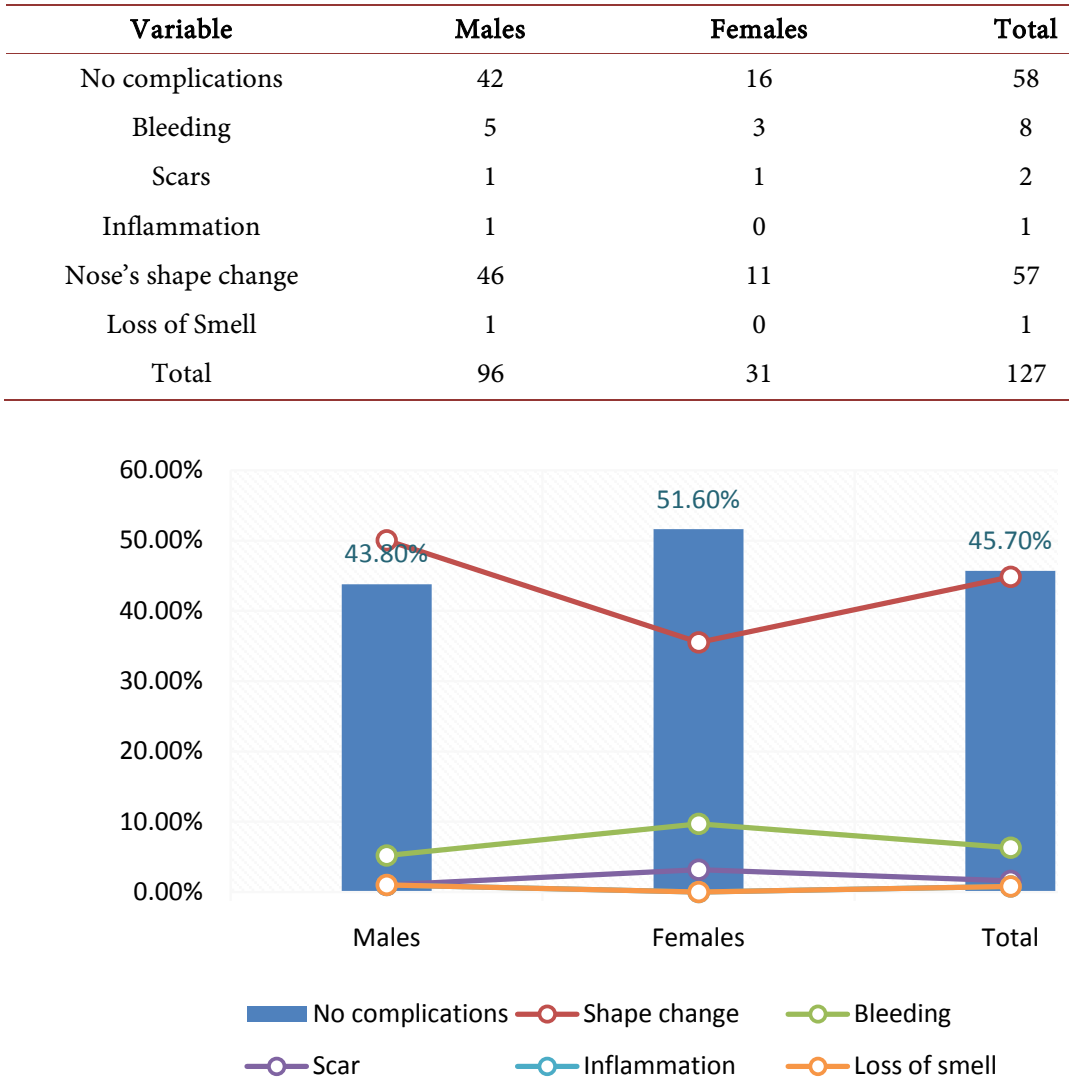

Figure 2. Description of patients by complications after three months of septoplasty.

Table 3. Distribution of patients by age, operation done and nasal blockage by complications after three months of septoplasty.

\begin{tabular}{|c|c|c|c|c|c|c|c|}
\hline Variable & NO & Change of shape & Bleeding & Scar & Inflammation & Loss of smell & Total \\
\hline \multicolumn{8}{|l|}{ Age } \\
\hline$<20$ years & 11 & 12 & 1 & 1 & 0 & 0 & 25 \\
\hline $21-25$ & 16 & 18 & 5 & 0 & 0 & 1 & 42 \\
\hline $26-30$ & 17 & 11 & 0 & 0 & 0 & 0 & 28 \\
\hline $31-35$ & 10 & 8 & 0 & 0 & 0 & 0 & 18 \\
\hline $36+$ & 4 & 8 & 2 & 0 & 1 & 0 & 14 \\
\hline Total & 58 & 57 & 8 & 1 & & 1 & 127 \\
\hline \multicolumn{8}{|l|}{ Option done } \\
\hline Septoplasty & 51 & 41 & 7 & 1 & 2 & 1 & 103 \\
\hline Septorhinoplasty & 7 & 16 & 1 & 0 & 0 & 0 & 24 \\
\hline Total & 58 & 58 & 8 & 1 & 2 & 1 & 127 \\
\hline \multicolumn{8}{|l|}{ Nasal Blockage } \\
\hline None & 25 & 28 & 2 & 1 & 0 & 1 & 57 \\
\hline Mild & 24 & 22 & 2 & 0 & 0 & 0 & 48 \\
\hline Moderate & 3 & 6 & 3 & 0 & 0 & 0 & 12 \\
\hline Severe & 6 & 1 & 1 & 1 & 1 & 0 & 10 \\
\hline Total & 58 & 57 & 8 & 2 & 1 & 1 & 127 \\
\hline
\end{tabular}




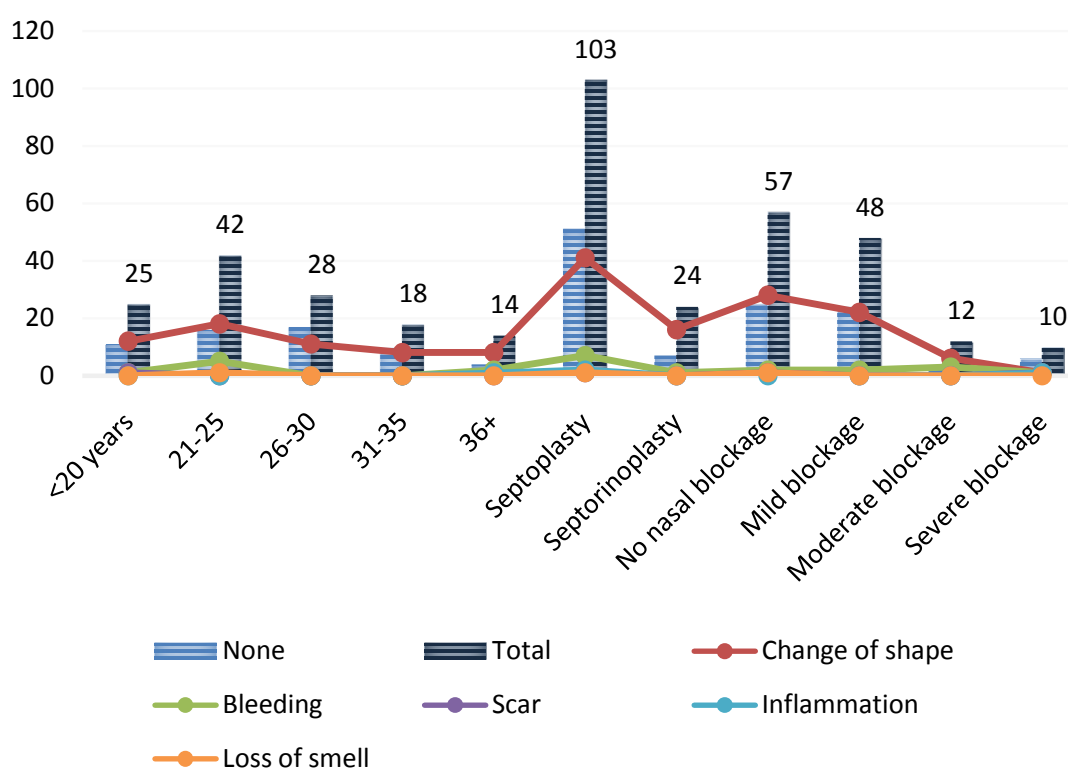

Figure 3. Description of patients by age, operation done and nasal blockage by complications after three months of septoplasty.

complications revealed multiple complications in $54.3 \%$ of the patients. Several studies have reported a diverse types of complications associated with Septoplasty or Septorhinoplasty [10] [11].

The most common complication after three months of Septoplasty or Septorhinoplasty was the change in the shape of the nose, which represented $82.6 \%$ out of the patients with complications. However most of complications depend on the septoplasty surgical procedure performed. Loss of caudal septal support appears to be a major reason for nasal deformity after septoplasty. Surgical correction for those deformities is a formidable task to deal with, because the septal cartilage framework was already weakened due to previous procedure [12]. Establishing a straight and firm septum supporting the overlying nasal structures is the most important step in correcting the post-traumatic combined deviated and saddle nose [13]. Moreover, postoperative deformities are considered as main risks of rhinoplasty, causing revision surgery in $5 \%$ to $15 \%$ of the cases [14]. The Polly beak is the indication in about $50 \%$ of all revision rhinoplasty. Other frequent postoperative deformities are a pendant and wide nasal tip, retractions of the columella base or irregularities of the nasal dorsum. These deformities are very often combined and caused by a loss of septal support. This is why the stability of the caudal septum in Septorhinoplasty is the key for a predictable result. Maintaining the position of the tip and the columella is one of the main issues to avoid typical postoperative deformities [15]. Knowledge and understanding of these dynamics and relationships of the nasal base and the surrounding structures is essential to minimize the risk for postoperative deformities.

In the present study bleeding was encountered in $11.6 \%$ of the patients with complications. Postoperative bleeding most commonly occurs within the first 24 hours of the procedure, but can be delayed days or even weeks. If the bleeding 
causes a hematoma (blood clot) within the septum, removal of the hematoma is necessary, and the development of scar tissue or even nasal collapse could occur [16] [17]. However, for persist for over 3 month this might be associated with other conditions.

In the current study scar was found among $2.9 \%$ of the patients with complications. Although, it represented small proportion, scar was regularly encountered in septoplasty [18] [19]. Other complications including inflammatory change and loss of smell were also experienced in this series of patients, which was previously reported in similar patients' settings [8].

Furthermore, Septoplasty or Septorhinoplasty is based on experience. Besides knowledge of literature and techniques a critical surgeon is a prerequisite for reliable results. Knowing the risks, mistakes should be identified and avoided in the future. A complication as cause of an undesirable result should only be assumed if there is no evidence of a mistake in patient selection, preoperative planning and operative technique.

\section{Conclusion}

The deformities are the most common complication associated with Septoplasty or Septorhinoplasty in Northern Saudi Arabia. The risks for Septoplasty or Septorhinoplasty complications can be condensed with accumulative experience. A prerequisite is persistent education and a deep distinction between complication and inaccuracy.

\section{References}

[1] Jessen, M. and Malm, L. (1997) Definition, Prevalence and Development of Nasal Obstruction. Allergy, 52, 3-6. https://doi.org/10.1111/j.1398-9995.1997.tb04876.x

[2] Sipilä, J. and Suonpää, J. (1997) Prospective Study Using Rhinomanometry and Patient Clinical Satisfaction to Determine If Objective Measurements of Nasal Airway Resistance Can Improve the Quality of Septoplasty. European Archives of Oto-Rhino-Laryngology, 254, 387-390. https://doi.org/10.1007/BF01642556

[3] Van Egmond, M.M.H.T., Rovers, M.M., Hendriks, C.T.M. and van Heerbeek, N. (2015) Effectiveness of Septoplasty versus Non-Surgical Management for Nasal Obstruction Due to a Deviated Nasal Septum in Adults: Study Protocol for a Randomized Controlled Trial. Trials, 16, 500. https://doi.org/10.1186/s13063-015-1031-4

[4] Manoukian, P.D., Wyatt, J.R., Leopold, D.A. and Bass, E.B. (1997) Recent Trends in Utilization of Procedures in Otolaryngology-Head and Neck Surgery. Laryngoscope, 107, 472-477. https://doi.org/10.1097/00005537-199704000-00009

[5] Kent, S.E., Reid, A.P., Nairn, E.R. and Brain, D.J. (1988) Neonatal Septal Deviations. Journal of the Royal Society of Medicine, 81, 132-135.

[6] Chintapatla, S., Kudva, Y.C., Nayar, R.C., Raghuveer, T.S. and Prasad, D. (1989) Septal Deviation in Neonates. Indian Pediatrics, 26, 678-682.

[7] Harugop, A.S., Mudhol, R.S., Hajare, P.S., Nargund, A.I., Metgudmath, V.V. and Chakrabarti, S. (2012) Prevalence of Nasal Septal Deviation in New-Borns and Its Precipitating Factors: A Cross-Sectional Study. Indian Journal of Otolaryngology and Head \& Neck Surgery, 64, 248-251. https://doi.org/10.1007/s12070-011-0247-1 
[8] Rettinger, G. and Kirsche, H. (2006) Complications in Septoplasty. Facial Plastic Surgery, 22, 289-297. https://doi.org/10.1055/s-2006-954847

[9] Foda, H.M. and Magdy, E.A. (2006) Combining Rhinoplasty with Septal Perforation Repair. Facial Plastic Surgery, 22, 281-288. https://doi.org/10.1055/s-2006-954846

[10] Ketcham, A.S. and Han, J.K. (2010) Complications and Management of Septoplasty. Otolaryngologic Clinics of North America, 43, 897-904. https://doi.org/10.1016/j.otc.2010.04.013

[11] Cochran, C.S. and Landecker, A. (2008) Prevention and Management of Rhinoplasty Complications. Plastic and Reconstructive Surgery, 122, 60e-7e. https://doi.org/10.1097/PRS.0b013e31817d53de

[12] Yeo, N.K. and Jang, Y.J. (2009) Rhinoplasty to Correct Nasal Deformities in Postseptoplasty Patients. The American Journal of Rhinology \& Allergy, 23, 540-545. https://doi.org/10.2500/ajra.2009.23.3344

[13] Won, T.B., Kang, J.G. and Jin, H.R. (2012) Management of Post-Traumatic Combined Deviated and Saddle Nose Deformity. Acta Oto-Laryngologica, 132, S44-S51. https://doi.org/10.3109/00016489.2012.659757

[14] Rettinger, G. (2007) Risks and Complications in Rhinoplasty. GMS Current Topics in Otorhinolaryngology, Head and Neck Surgery, 6, Doc08.

[15] Rettinger, G. (1997) Foreword Complication or Mistake? Facial Plastic Surgery, 13, 1. https://doi.org/10.1055/s-2008-1064460

[16] Mane, R.S., Patil, B. and Mohite, A. (2013) Comparison of Septoplasty with and Without Nasal Packing and Review of Literature. Indian Journal of Otolaryngology and Head \& Neck Surgery, 65, 406-408. https://doi.org/10.1007/s12070-013-0626-x

[17] Bajwa, S.J.S., Kaur, J., Singh, A., Parmar, S.S. and Singh, S. (2013) Postoperative Airway Management after Nasal Endoscopic Sinus Surgery: A Comparison of Traditional Nasal Packing with Nasal Airway. Anesthesia, Essays and Researches, 7, 116-122. https://doi.org/10.4103/0259-1162.114017

[18] Matthias, C. (2007) Surgery of the Nasal Septum and Turbinates. GMS Current Topics in Otorhinolaryngology, Head and Neck Surgery, 6, Doc10.

[19] Simon, P. and Sidle, D. (2012) Augmenting the Nasal Airway: Beyond Septoplasty. American Journal of Rhinology \& Allergy, 26, 326-331. https://doi.org/10.2500/ajra.2012.26.3786 